\title{
La Dematerialisation : Determinant Primordial De L'efficacite De La Commande Publique
}

\begin{abstract}
Abdelkader El Amry, (doctorant)
Université Ibn Tofaïl /

Faculté des Sciences Juridiques Economiques et Sociales -Kenitra, Maroc

Doi:10.19044/esj.2018.v14n19p63 URL:http://dx.doi.org/10.19044/esj.2018.v14n19p63

Abstract

In Morocco, public procurement represents $17.4 \%$ of GDP, equivalent to 195 billion dirhams in 2018 (Maghreb Arab Press, 2018), which makes it more exposed to unhealthy practices that can be engaged both by officials and operators across all stages of the procurement process. Above all, such practices create a climate devoid of transparency and generates weaknesses in public procurement systems. It is, therefore, essential for the Moroccan administration to integrate anti-corruption measures into the procurement process. Among these anti-corruption measures, there is the " e-procurement " which all specialists in the field of public control unanimously assume that it has become key in the promotion of competition, transparency, integrity, simplification of procedures, and modernization of public expenditure execution processes. Moreover, they admit that it contributes to a large extent to reduce corruption without destroying it. To inquire about the veracity of the contribution of the e-procurement to the efficiency of public order, a questionaire was administered to four co-ordinators and four companies of different working fields in the city of Meknes. The objective is to explore the relationship between e-procurement and the fundamental principles of public control. Eventually, the results of this study revealed that eprocurement is determinant to the effectiveness of public procurement.
\end{abstract}

Keywords: Efficiency, E-procurement, Transparency, Competition, professionalization

\section{Résumé}

$\mathrm{Au}$ Maroc, la commande publique représente 17,4\% du PIB soit l'équivalent 195 MMDH en 2018 (Maghreb Arabe Presse. 2018), ce qui la rend plus exposée à des pratiques malsaines qui peuvent être engagé à la fois par le fonctionnaire et par l'opérateur, dans toutes les étapes du processus de passation du marché, chose qui engendre une procédure dépourvue de la 
transparence et génère des faiblesses dans les systèmes de passation de marchés publics. Il est donc primordial pour l'administration marocaine d'intégrer des mesures de lutte contre la corruption dans la procédure de passation des marchés publics. Parmi les mesures anti-corruption, on trouve la « dématérialisation » que tous les spécialistes en domaine de la commande publique sont unanimes que cette dernière est devenue l'élément clé qui permet de promouvoir la concurrence, la transparence, l'intégrité, la simplification des procédures, la modernisation des processus d'exécution de la dépense publique et contribue dans une large mesure à réduire la corruption sans l'anéantir. Pour s'enquérir sur la véracité de l'apport de la dématérialisation à l'efficacité de la commande publique. Une enquête sur le terrain a été réalisée sous forme d'un questionnaire déposé successivement auprès de 04 sous ordonnateurs et auprès de 04 entreprises, de différentes catégories, exerçant dans le domaine des travaux relevant de la ville de Meknès. L'objectif étant de s'enquérir sur le rapport liant la dématérialisation et les principes fondamentaux de la commande publique. Le résultat de l'enquête a révélé que la dématérialisation est un déterminant primordial de l'efficacité de la commande publique.

Mots clés: L'Efficacité, La Dématérialisation, La Transparence, La Concurrence, La professionnalisation

\section{Introduction :}

La dématérialisation de la commande publique est devenue, aujourd'hui, une réalité universelle très partagée. Elle occupe une place prépondérante dans les politiques publiques, la modernisation, la simplification des démarches et procédures administratives de tous les pays.

Le domaine des marchés publics, comme celui de certaines concessions des services publics, reste très exposé à la corruption, et entache 1 image de certains pays, qui font invariablement l'objet de critiques et protestations (EL Mesbahi, 2008). Et ce, a cause de leur mauvaise gestion des marchés publics, marquée par la fraude, la corruption.

Selon Dabchy (2015), en dépit de la réglementation et des contrôles existant dans la commande publique, des tares entacheraient encore un certain nombre de marchés publics.

En effet, le nombre des affaires liées aux détournements de fonds à origine publique au profit des intérêts privés, d'individus ou d'organisations, constamment révélées par les médias, cesse d'augmenter. Les méthodes frauduleuses utilisées par les fonctionnaires et les entrepreneurs indélicats portent généralement sur les trois phases des marchés publics ci-après:

Phase préalable à la décision :Les mécanismes frauduleux, utilisés avant le lancement de l'appel d'offre, peuvent concerner aussi bien une 
estimation surévaluée du marché, la multiplication des études de complaisance, l'insertion des prestations inutiles au niveau du CPS, le fractionnement des marchés publics, un cahier des charges sur mesure pour privilégier un soumissionnaire, des imprévus de chantiers volontairement dissimulés mais annoncés discrètement à un soumissionnaire complice qui, lui, en tiendra compte, dans son offre financière etc.

Lors du lancement de l'appel d'offre: L'une des fraudes les plus courantes est l'entente de marchés. Il s'agit pour les entreprises consultées de se mettre d'accord, d'une manière occulte, sur l'entreprise parmi elles qui obtiendra le marché. Les concurrents, qui sont volontairement écartés de la signature du contrat, obtiennent des contreparties (attribution d'un autre marché, partage des bénéfices, sous-traitance..).

Pendant la phase de l'exécution du marché : Lorsque l'on entre dans la phase de réalisation du marché, on est le plus souvent confronté à des prestations matérielles non réalisées, mal faites ou de qualité inférieure à celles prévue, du travail clandestin, des surveillants et des contrôleurs complices de détournements, outre un ensemble de pratiques profondément intégrées dans la tradition du métier concerné ,comme les travaux supplémentaires surfacturés à cause de la multiplication des avenants ou des marchés attribués à des entreprises en relation indirecte avec le fonctionnaire. Tous ces artifices évoqués par Dabchy permettent au titulaire du marché de générer les flux financiers nécessaires à la réalisation du pacte corrupteur. Il s'agit de réduire la qualité des prestations imposée au fournisseur pour lui permettre ainsi de générer davantage de profit et de verser une commission au décideur.

Chacune de ces méthodes est susceptible de camoufler des détournements de fonds publics ou des phénomènes de corruption, trafic d'influence, concussion, prise illégale d'intérêts, atteinte à la liberté et à l'égalité des candidats, tout en donnant une apparente régularité aux divers stades de la procédure.

Si le phénomène de la fraude dans les marchés publics n'est pas efficacement combattu, certes, les conséquences économiques, sociales et politiques seront désastreuses. Les citoyens sont, en effet, les principales victimes de ces agissements malhonnêtes : victimes sur le plan fiscal (par l'augmentation injustifiée de la pression fiscale puisqu'il faut plus d'argent pour réaliser un projet), victime sur le plan économique (la disparition des entreprises qui refusent ou ne peuvent payer les pots-de-vin réclamés par les décideurs corrompus) et victimes sur le plan social (chômage et exclusion, insuffisance des infrastructures de base, affablement de la solidarité et des valeurs morales et, avec elles les fondements de la société démocratique).

A cet effet, le recours au levier de l'économie numérique «dématérialisation » dans le domaine de la commande publique 
paraît comme un outil fondamental pour changer la donne dans la relation de l'administration avec les entrepreneurs, fournisseurs et prestataires.

En effet, le recours au levier numérique est non seulement un accélérateur puissant dans l'amélioration du service au citoyen, mais également un moyen très efficace de lutte contre les pratiques illicites de corruption ou autres abus de pouvoir qu'une administration peut exercer, du simple fait, qu'elle détient une information que le citoyen n'a pas.

$\mathrm{Vu}$ ce qui précède, notre article se focalisera sur le volet suivant : «comment la dématérialisation constitue-elle un déterminant primordial de l'efficacité de la commande publique».

\section{Aperçu Général sur la Dématérialisation de la Commande Publique}

Selon le Ministère de l'Economie et des Finances (2017), la réforme budgétaire au Maroc lancée à partir de 2001 s'inscrit au cœur d'un vaste programme de modernisation de l'administration publique. Elle a été accompagnée par le développement des systèmes d'information intégrés de gestion budgétaire qui visent la dématérialisation et la mutualisation de l'information budgétaire. Il s'agit, pour la programmation budgétaire, du système "e-budget" qui a pour objectif de mettre en ligne les services de prise en charge, d'analyse et de suivi des opérations budgétaires au profit des départements ministériels; et pour l'exécution budgétaire du système "Gestion Intégrée de la Dépense" (GID) qui a notamment pour objectifs, l'accélération du traitement des actes liés à la dépense, la rationalisation, la simplification des circuits et des procédures d'exécution des dépenses publiques. Et c'est dans ce cadre que s'inscrit l'apparition de la dématérialisation.

Selon la définition du Ministère de la réforme de l'Administration et de la fonction publique (2016) « La dématérialisation a pour but le remplacement de l'écrit papier par l'écrit électronique et de favoriser les échanges d'information par des moyens de communication informatiques aboutissant à des gains de productivité et de qualité de service ». Concrètement, il s'agit de scanner des documents clés pour l'entreprise, afin d'en avoir une copie électronique, plus facilement copiable, transférable et emmagasinable.

La dématérialisation de la commande publique au Maroc est un processus qui a connu des évolutions notoires au cours des dernières années, grâce à la disponibilité d'un arsenal de textes juridiques, bien déterminés et réglementés par les quatre principaux textes de référence ci après énumérés :

- Loi $n$ ○ 53-05 relative à l'échange électronique de données juridiques promulgué par le Dahir $\mathrm{n}^{\circ}$ 1-07-129 du 19 kaada 1428 (30 novembre 2007).

- Décret $\mathrm{n}^{\circ}$ 2-08-518 pris pour l'application des articles $13,14,15$, 21 et 23 de la loi $n^{\circ} 53-05$ relative à l'échange électronique des données juridiques. 
- Chapitre VII du décret n ${ }^{\circ} 2-12-349$ du 20 mars 2013 relatif aux marchés publics (articles 147 à 151). • Arrêté $\mathrm{n}^{\circ} 1872-13$ du 13 juin 2013 relatif aux modalités de publication sur le portail des marchés publics.

- Arrêté $\mathrm{n}^{\circ} 20-14$ du 4 septembre 2014 relatif à la dématérialisation des procédures de passation des marchés publics.

La mise en place de la dématérialisation au Maroc est le fruit d'une concertation approfondie entre les différents intervenants de la commande publique, qui vise les objectifs stratégiques illustrés ci-dessous :

- Assurer la transparence dans la gestion des marchés publics;

- Garantir le libre jeu de la concurrence dans l'attribution des marchés ;

- Inciter à la performance dans la préparation et l'exécution des commandes publiques;

- Garantir les droits des entreprises soumissionnaires;

- Simplifier les procédures et les règles régissant la gestion des marchés publics;

- Réduire le contact physique entre les administrations et le citoyen, éliminer les privilèges, le clientélisme et renforcer le contrôle ;

- Garantir la traçabilité de toutes les étapes et décisions (y compris les réclamations et leur traitement) avec un reporting riche et rigoureux ;

- Permettre un large accès à l'information en temps opportun et à tous moment ;

- Contribuer au professionnalisme des cadres en la matière ;

- Contrôler avec fiabilité la procédure de passation de la commande publique ;

- Contribuer à l'éthique et à la moralisation des acheteurs publics (décret susvisé ${ }^{\circ}$ 2-12-349 du 8 joumada 11434 (20 mars 2013).

En sus, et grâce aux exigences de modernisation de la commande publique, le Maroc s'est trouvé dans l'obligation d'adopter la dématérialisation comme nouveau instrument indispensable au mouvement de généralisation des échanges dématérialisés, aussi bien entre personnes qu'entre personnes et entreprises et/ou administrations ou encore entre entreprises.

En relation avec le sujet, Moutenet, Mechalia, Bonnefoy, Eksl, Héraud \& Passemard, (2011), spécialistes du domaine ont considéré la dématérialisation comme une plateforme qui a contribué à :

- La convergence des moyens de communication de la voix, de l'image, des données qui empruntent tous aujourd'hui des réseaux informatiques ; 
- La (relative) facilité d'usage pour la grande majorité des clients, des citoyens, des consommateurs quand on remplace les échanges papier par des communications électroniques ;

- La rapidité des échanges qu'il s'agisse d'informations générales ou professionnelles (sur les sites internet ou intranet) ; d'échanges interpersonnels ou des échanges de données de gestion ;

- Les économies qui résultent le plus souvent de l'utilisation de ces techniques, économies qui contribuent à la baisse des prix de revient dans la mise en œuvre des services et à une meilleure performance des entreprises et des administrations ;

- un souci de développement durable (une moindre consommation de papier puisque le nombre de pages papier économisées est considérable et de $\mathrm{CO} 2$ enfin avec la réduction des dépenses d'énergie liées au transport des plis et des documents).

\section{La Dématérialisation un Moyen Efficace de Contrôle des Marchés Publics}

Vu l'importance des marchés publics en termes financiers qui atteint 195 MMDH en 2018, soit l'équivalent de $17,4 \%$ du PIB et en termes stratégiques (acquisition des biens et des services et réalisation des travaux dont l'administration a besoin), le gouvernement marocain a progressivement pris conscience du risque de la corruption dans les marchés publics et de la nécessité de la bannir voire la prévenir. A cet effet, il a fait recours au déploiement de la plateforme de dématérialisation qui permet aux services gestionnaires d'assurer leurs prérogatives dans les meilleures conditions de célérité, de fiabilité et de sécurité requises.

De plus, la dématérialisation joue un rôle primordial dans le contrôle de toutes les opérations administratives, budgétaires et comptables des ordonnateurs ou des sous-ordonnateur, notamment :

- Le contrôle efficace des opérations administratives à travers la traçabilité de toutes les transactions effectuées dans toutes les étapes de déroulement de la procédure des dossiers d'appels d'offres au niveau du système de gestion intégrée de la dépense par le biais d'archivage sécurisé.

- Le contrôle de la qualité de l'ordonnateur ou du sous-ordonnateur et celle de leurs délégués ou suppléants ;

- Le contrôle de l'existence des rubriques budgétaires concernées ;

- Le contrôle de la disponibilité des crédits ;

- Le contrôle de la disponibilité des recettes pour les comptes spéciaux

- La mise à jour automatique de la comptabilité budgétaire ; 
- Le respect des seuils des dépenses tels que prévus par les textes régissant le contrôle des dépenses de l'Etat. (Trésorerie Générale du Royaume, 2009)

\subsection{Les Organismes de Contrôle des Marchés Publics}

Dans le cadre d'optimisation et rationalisation de la gestion de la commande publique, la loi marocaine a soumis cette dernière à certains modes de contrôle exercés par des organismes divers pour mieux encadrer l'opération des marchés publics.

En effet, le contrôle dans le cadre de la commande publique est une fonction qui consiste à suivre un marché public depuis sa conception jusqu'à sa réalisation finale.

La fonction de contrôle est importante dans la mesure où elle permet ou plutôt aide à passer sans grande entorse de la phase des idées à celle de la réalisation concrète du marché.

Cet aperçu général sur la fonction de contrôle nous amène à nous interroger sur les les organismes de contrôle de l'administration et leurs mécanismes.

\subsubsection{Le Contrôle Administratif}

Le contrôle administratif est pratiqué par l'ensemble des instruments d'intervention mobilisé par l'administration en amont et en aval de la dépense publique. (Hanine, 2007)

C'est un contrôle qui peut être effectué lors de la passation des contrats, durant son exécution, au moment du paiement, et peut intervenir aussi en tant qu'instance de règlement des différends à l'amiable.

Il s'agit principalement du contrôle des engagements de dépenses, du contrôle de l'inspection générale des finances et du contrôle de la commission nationale de la commande publique.

\subsubsection{Le Contrôle Juridictionnel}

Selon Hanine (2007), il s'agit d'un contrôle exercé par les juridictions financières, se définit par sa matière constituée des finances publiques. Le contrôle supérieur des finances publiques relève au Maroc de la cour des comptes et des cours régionales des comptes. Ce contrôle se distingue du contrôle exercé par d'autres organes administratifs de part le statut constitutionnel de la cour des comptes, qui lui confère les attributs d'organe supérieur de contrôle des finances publiques.

En somme, la finalité escomptée de ces deux types de contrôle dans le domaine de la commande publique est:

- La mise en place d'un système transparent, efficace et équitable dans la passation des marchés. 
- L'appréciation de l'efficacité de la réalisation commandes publiques, l'amélioration et l'adéquation constante des objectifs tracés et les moyens mis en œuvre.

Malgré tous les arguments étalés à travers cet article, justifiant la mise en place de la plateforme d'échange dématérialisé, l'avis en l'objet des entreprises et des administrations marocaines demeure encore inconnu. Dans le souci de surpasser cette situation, la partie empirique de notre étude dont le résultat récapitulé ci-dessous, tente de rapporter tant bien que mal quelques avis et opinions des dits milieux.

\section{Etude Empirique sur la Dématérialisation de la Commande Publique}

Le questionnaire proposé dans cette étude vise à confirmer, ou infirmer voire évaluer : comment la dématérialisation de la commande publique adoptée dans le nouveau décret constitue-elle un déterminant primordial de l'efficacité de la commande publique ?

Avant tout, il convient d'évoquer que l'efficacité regroupe quatre principes indispensables et indissociables qui sont comme suit :

H1 : La dématérialisation de la commande publique

H2: La professionnalisation des acheteurs publics

H3 : La transparence

H4 : Le libre jeu de la concurrence

L'ordre de classement de ces hypothèses n'est pas arbitraire, mais il est mu par l'importance de chaque phase. En effet, la dématérialisation est un élément qui illustre la détermination du pays à assurer la transparence et à lutter contre la corruption. De même, elle constitue -à notre avis - la méthode la plus efficace pour accroître la transparence, la concurrence et la contribution dans une large mesure au professionnalisme des cadres en la matière en leur permettant de mieux gérer et de maîtriser les techniques informatiques de cette plateforme.

Pour s'assurer de la fiabilité des quatre hypothèses précitées, entant que déterminants indispensables à l'efficacité de la commande publique, une enquête sur le terrain a été menée sous forme d'un questionnaire déposé successivement auprès de 04 sous ordonnateurs jugés qualifiés dans le domaine de dématérialisation entant qu'acheteurs publics . De même, 04 entreprises de différentes catégories exerçant dans le domaine des travaux de marchés publics entant que des vendeurs relevant de la ville de Meknès, ont été interpellées et invitées à s'exprimer sur l'apport de la dématérialisation de la commande publique à l'efficacité de la commande publique. Par ailleurs ces mêmes échantillons ont été sollicités pour donner leurs avis sur la relation entre la dématérialisation (H1) et les autres déterminants à savoir la professionnalisation des acheteurs publics (H2), la transparence (H3), le libre 
jeu de la concurrence $(\mathbf{H 4})$, et comment ils contribuent en commun à l'efficacité de la commande publique.

En somme, le résultat de l'enquête empirique a révélé les réponses figurées dans le tableau ci-dessous :

Tableau 1. Matrice de passage relative à la dématérialisation de la commande publique

\begin{tabular}{|c|c|}
\hline Les questions & $\begin{array}{l}\text { Les réponses }(\%) \\
\text { (La moyenne) }\end{array}$ \\
\hline H1) La dématérialisation est-elle un moyen d'efficacité de la commande publique? & $100 \%$ Oui \\
\hline $\begin{array}{l}\text { H2) Est-ce que la dématérialisation renforce-elle la professionnalisation des } \\
\text { acheteurs publics? }\end{array}$ & $100 \%$ Oui \\
\hline $\begin{array}{l}\text { H3) Comment la dématérialisation assure-t-elle la transparence de la commande } \\
\text { publique? }\end{array}$ & $100 \%$ Oui \\
\hline H4) Est-ce que la dématérialisation promeut-elle le libre jeu de la concurrence ? & $100 \%$ Oui \\
\hline $\begin{array}{l}\text { H1/H2/H3/H4 : A votre avis, la dématérialisation, la professionnalisation des } \\
\text { acheteurs publics, la transparence et la concurrence, } \\
\text { constituent-elles en commun des déterminants incontournables de l'efficacité de la } \\
\text { commande publique? }\end{array}$ & $100 \%$ Oui \\
\hline
\end{tabular}

D'après l'analyse des réponses obtenues suite aux questions illustrées dans le tableau ci-dessus, il appert que la dématérialisation (H1) constitue effectivement un déterminant incontournable de l'efficacité de la commande publique, tous les sous ordonnateurs et les entreprises touchés par cette enquête ont été unanimes à affirmer que la dématérialisation a incontestablement simplifié le travail tant pour les acheteurs publics que pour les entreprises soumissionnaires:

\section{Pour les entreprises soumissionnaires}

- La Simplification des procédures des marchés publics ;

- La réduction des coûts de prospection des marchés publics ;

- L'accès rapide à l'information et en temps opportun ;

- Le renforcement de la transparence et de la lutte contre la corruption;

- L'accès unique et centralisé à l'ensemble des appels à la concurrence;

- La mise en concurrence loyale à travers droit d'accès à une information exhaustive et structurée sur les marchés publics;

- La simplification de la procédure de soumission aux marchés publics;

- La recherche sélective des opportunités de marchés ;

- La disponibilité d'une base de donner des textes réglementaires et juridiques constamment mise à jour; 
- La disponibilité d'un annuaire des acheteurs publics.

\section{Pour les acheteurs publics}

- Contribuer au professionnalisme des cadres en la matière ;

- La maîtrise des délais de publication ;

- les appels d'offres peuvent désormais être annoncés en deux minutes

- Une économie grâce à une concurrence accrue et élargie ;

- Une facilité dans les procédures de modification des dossiers de consultation et de réponse aux demandes d'éclaircissements ;

- La disponibilité d'une base de donner des textes réglementaires et juridiques constamment mise à jour ;

- La disponibilité d'un espace communautaire pour le partage des connaissances (Forums, Foire Aux Questions...) ;

- L'assistance dans la passation de la commande publique (CPS types, formulaires) ;

- La disponibilité d'une base de donner des fournisseurs etc.

De plus, tous les sous ordonnateurs et les entreprises enquêtées ont été unanimes que la dématérialisation (H1) constitue un moyen inévitable pour la réalisation des autres hypothèses à savoir : la professionnalisation des acheteurs publics (H2), la transparence $(\mathbf{H 3})$ et la mise en concurrence $(\mathbf{H 4})$, et que ces quatre déterminants contribuent en commun à l'efficacité de la commande publique.

\section{Conclusion:}

Bien que la dématérialisation des marchés constitue une avancée considérable dans la gestion de la commande publique. Et aussi prometteuse que soit elle, cette technique comporte quelques incertitudes compte tenu de l'évolution rapide des outils indispensables à sa mise en place :

- La réactualisation des outils : concerne aussi les moyens de protection contre les menaces venant du réseau. A défaut, le risque encouru est celui de l'obsolescence des outils à court terme.

- Le danger du piratage informatique : constitue une véritable menace pour les données car les risques de pertes ou de corruption de ces données est énorme. C'est pourquoi, les moyens de protection des systèmes doivent être sûrs et continuellement mis à jour.

- Le risque de virus informatiques : les virus représentent une véritable menace pour les systèmes informatiques, du fait qu'ils pourraient perturber plus ou moins gravement le bon déroulement des procédures dématérialisées d'attribution des marchés publics, empêcherait la lecture par la commission d'ouverture des plis et causerait le rejet de certaines offres pour 
défaillance technique du système ce qui entraverait le principe d'égalité de traitement des candidats.

\section{References:}

1. Dabchy, H. (2015). Les fraudes dans les marches publiques. Revue de Police, 7, 38-39.

2. Décret susvisé $n^{\circ}$ 2-12-349 du 8 joumada 11434 (20 mars 2013).

3. EL Mesbahi, K. (2008). Transparence versus Transparence versus corruption dans les marchés publics : lecture à travers les dispositions de l'Accord de libre-échange Maroc / Etats-Unis. Critique économique, 21, 143-159.

4. Hanine, M. (2007). La procédure de passation des marchés publics au Maroc : étude analytique et réflexions à la lumière du code français des marchés publics (et des directives européennes) et des directives de la Banque Mondiale (Thèse de Master). Ecole Nationale D’Administration, Paris, France.

5. Hors, I. (2000). Les difficultés de lutte contre la corruption. Revue Tiers Monde, 161,143-163.

6. Maghreb Arabe Presse. (2018). Installation de la commission nationale de la commande publique. Extrait de http://www.maroc.ma/fr/actualites/installation-rabat-de-lacommission-nationale-de-la-commande-publique

7. Ministère de l'Economie et des Finances. (2017). La Loi Organique relative à la loi de Finances - Maroc «Genèse et évolution ». Extrait de http://lof.finances.gov.ma/printpdf/320.

8. Ministère de la réforme de l'Administration et de la fonction publique. (2016). Commission Nationale Anti-corruption (CNAC) «Stratégie Nationale de Lutte Contre la Corruption». Extrait de https://www.mmsp.gov.ma/uploads/file/Strategie\%20Nationale\%20d e\%20lutte\%20Corruption_SNLCC_FR_2016.pdf

9. Moutenet, P., Mechalia, M., Bonnefoy, M.A., Eksl, R., Héraud, D., \& Passemard, P. (2011). Etude des impacts de la dématérialisation des données et des échanges sur les métiers et les compétences au sein des organismes de sécurité sociale. Extrait de http://extranet.ucanss.fr/contenu/public/EspaceUcanss/les_rapports/p df_html/etudes/cpnefp/rapport_phase_4_synthese_etude.pdf

10. Programme de Dématérialisation de la Commande Publique (PDCP). (2018). Plate-forme d'échange commune entre les acheteurs publics et les fournisseurs. Extrait de https://www.marchespublics.gov.ma/pmmp/spip.php?rubrique31\&la $\mathrm{ng}=\mathrm{fr}$ 
11. Trésorerie Générale du Royaume. (2009). Circulaire relative à la dématérialisation de certains actes de gestion budgétaire et comptable dans le cadre $d u$ système GID. Extrait de https://www.tgr.gov.ma/wps/wcm/connect/2fcbcc1d-98b6-4aa7-b7e6 c1968599e837/dematrialisation\%2Bde\%2Bcertains\%2Bactes\%2BBC \%2Bdans\%2Ble\%2Bcadre\%2Bsystme\%2BGID.pdf?MOD=AJPERE $\underline{\mathrm{S} \& C A C H E I D=2 \text { fcbcc1d-98b6-4aa7-b7e6-c1968599e837 }}$ 